\title{
A Study of the Adsorption and Diffusion Behavior of a Single Polydimethylsiloxane Chain on a Silicon Surface by Molecular Dynamics Simulation
}

\author{
Dan $\mathrm{Mu}^{1}$ and Jian-Quan $\mathrm{Li}^{2}$ \\ ${ }^{1}$ College of Chemistry Chemical Engineering and Materials Science, \\ Zaozhuang University, Shandong \\ ${ }^{2}$ Photoelectric Engineering College, Zaozhuang University, Shandong
}

China

\section{Introduction}

In three dimensions, polymer dynamics exhibits a rich and complex behavior which depends on the solvent conditions and polymer concentration $(1 ; 2)$. That the dynamics of polymer chains at and near solid interfaces differs profoundly from that in the bulk is intuitively expected. Polymer adsorption on the surface is of technological and scientific importance in the field of colloids and biomolecules. Examples include the two-dimensional (2-D) diffusion of DNA oligonucleotides confined to biological interfaces such as cell membranes (3; 4). The diffusion of confined polymers at surface is always a fundamental, yet problematical topic in polymer physics $(1 ; 2 ; 5-8)$. The behavior of polymers at the liquid-solid interface is crucial to technologies involving molecular surface placement $(9 ; 10)$. Polymers adsorbed onto a surface to form thin films is an emerging topic of modern materials science $(11 ; 12)$. They can be applied, for example, in the fields of biosensors, light-emitting diodes, nonlinear optical devices, and permeation-selective gas membranes (13-18). The fabrication of the thin films always takes place in vacuum or dilute solutions. The adsorption of the polymer chains can be controlled by varying multiple parameters such as the polymer-surface interaction, the solvent quality, surface nano-roughness, temperature, polymer chain length and so on. The knowledge about the adsorption dynamics and the thermodynamics of the equilibrium adsorption is crucial to understand and furthermore improve the property of the final product. Experimentally it is difficult to control well the above influences separately, thus, clear information on the exact effect of a specific environmental parameter is hard to obtain. Due to the fast-growing computation power nowadays, it is possible to utilize the computer as an "experimental apparatus" to solve the problem, with the aid of various simulation techniques. It has attracted attention for decades already.

Granick and co-workers studied poly(ethylene glycol) molecules adsorbed on solid surface by means of fluorescence microscopy (19-22). They found that the diffusion coefficient $(D)$ of such chains scales with the degree of polymerization $(N)$ as $N^{-3 / 2}$, which is characteristic for 
2-D chain models. On the other hand, Maier and Rädler found much weaker scaling, namely $N^{-1}$, when studying adsorbed DNA in a lipid bilayer (3; 4). In the simulations, Milchev and Binder (23) showed that $D$ scales with the chain length as $N^{-1.1}$; Azuma and Takayama (24) obtained $D \sim N^{-3 / 2}$; but Falck et al. (25) found that $D$ should scale as $N^{0}$. Therefore, the dynamics behavior of a confined polymer on a surface is still an interesting research topic.

Computer simulations for the mechanism of a single polymer chain adsorbed and diffusing on a surface are important, as experimental studies on an isolated polymer chain are difficult in most cases. Many simulations had been performed for different polymeric systems with multiple chains (or a single chain) adsorbing and diffusing on the surface (24-27).

Binder and coworkers successfully studied polymer films in different conditions via computer simulations (28-31). Lu and Kim observed the surface pattern of a thin polymer film by using a three-dimensional electrostatic model. The competition between the electrostatic energy and the surface energy leads to a characteristic pillar size. Furthermore, the film thickness significantly influences the growth rate and the distance between pillars (32). Recently, Kumar and coworkers found that a non-wetting solvent aids adsorption of the polymer chain at low sticking energies compared to wetting solvent by the aid of molecular dynamics simulations (33). However, there was no literature about the adsorption and diffusion of the PDMS (polydimethylsiloxane) single chain on the silicon surface. In this research, our main objective is to study the adsorption and diffusion processes of a hydrophobic chain on a hydrophobic surface. The similar hydrophobic-hydrophobic systems usually appears in the wetting, surface adhesion and flow in confined geometries are examples of such systems. Because we have completed some research about the poly (vinyl alcohol) adsorbing on the hydroxylated $\beta$-cristobalite (34), polyethylene chain adsorbing on the hydroxylated $\beta$-cristobalite (35), and the polyethylene chain adsorbing on the silicon (111) surface (36), then the results of the polydimethylsiloxane adsorbing on the silicon (111) surface may be helpful and enriching for further understanding of the configuration change and the dynamics of the hydrophobic polymer chain adsorbed on a hydrophobic surface.

We consider a single PDMS chain adsorbed on the rigid (111) silicon surface in a vacuum and in mimetic good solvent condition. The adsorption and diffusion processes of the chain are investigated through energy minimizations and molecular dynamics simulations with all the molecular degrees of freedom being taken into account. Only one single chain is adopted in a simulation because it relates directly to 2-D polymer diffusion in very dilute solution. In addition, PDMS is a widely available polymeric material, comes in a range of molecular weights, and is easy to use. The hydrophobic silicon is chosen because of its relative simplicity and rigidity, so it can be treated as a fully rigid body for our purposes. We follow a two-step strategy: first, we carry out direct energy minimizations of the PDMS chain close to the silicon surface with different initial configurations in order to relax the model; second, we use the most stable configuration after minimization to perform MD (Molecular Dynamics) simulation. In the theory of dynamical scaling the two key quantities are the radius of gyration $\left(R_{g}\right)$ and the center-of-mass diffusion coefficient $D$ of the chain. We thus use them to describe the characteristics for the system in this paper. We also change the effective dielectric constant to 78.0 to mimic good solvent condition. The results are compared with the systems in a vacuum (which can be taken as in bad solvent condition). The chain configurations show 
similar, but the diffusion coefficients vary greatly, showing strong dependence on the solvent condition.

\section{Models and simulation details}

The MD simulation is carried out in a box with 3-D periodic boundary conditions. The PDMS chain is embedded into the simulation box with a fixed (111) silicon surface parallel to the $X Y$ plane. We choose $N$ for the chain as 10, 20, 30, 40, 50 and 60. The thickness of the surface is around $12 \AA$. The length of the simulation box in the $Z$ direction is $80 \AA$, which is large enough so that the interactions between the adsorbed PDMS chain and the periodic images of silicon in the top plane can be ignored. In this way, the 3-D periodicity inherent in the model is transformed into an actual 2-D periodicity thus simulating an infinitely extended surface.

A high-quality force field COMPASS (condensed-phase optimized molecular potentials for atomistic simulation studies) $(37 ; 38)$ is adopted in the simulation. In contrast to early force fields which were mostly parameterized based on gas-phase data or ab initio calculations, COMPASS combines ab initio and empirical parametrization procedures. In addition, it adds cross terms to potential in order to consider the influence of all atoms close-by and distortions of bond length or bond angle. It enables accurate and simultaneous prediction of structural, conformational, vibrational and thermophysical properties for a broad range of molecules in isolation and in condensed phases. The energy calculation with COMPASS is a combination of bonding and non-bonding terms. The bonding terms include stretching, bending and torsion energy as well as the diagonal and off-diagonal cross coupling terms. The van der Waals interactions are truncated at $r_{c}=12 \AA$ by using a spline function from $11 \AA$. The Coulomb interactions are calculated via Ewald summation (39). Before the MD simulations, energy minimizations are performed to relax the local unfavorable structure of the chain. Subsequently, MD simulations with $5 \mathrm{~ns}$ are performed under NVT thermodynamics ensemble. Every simulation is performed three times to ensure the reliability of the results. The equations of motion are integrated with a time step of $1 \mathrm{fs}$. The constant temperature $T=300 \mathrm{~K}$ is controlled through the Berendsen thermostat (40) with a relaxation time of $0.1 \mathrm{ps}$.

In these simulations, the total and potential energies show an initial decrease, possibly with a few separate kinetic stages, and then fluctuate around a constant value, indicating the achievement of the equilibrium state. This process corresponds to the adsorption and diffusion dynamics of the PDMS chain from the initial configuration. We then change the dielectric constant to 78.0 to mimic the good solvent condition. Of course in this way the explicit solvation effects cannot be considered. Nevertheless, we can directly study the effects by simply changing the bad to the good solvent condition on the chain configurations and dynamics.

\section{Simulation results and discussion}

In this section, we show the simulation results, such as the chain configurations, the diffusion coefficient and the adsorption energy. All simulations are run until the chain reaches its equilibrium structure, i.e., until the simulated chain lost its memory of the initial configuration, and running the program further results in no discernible changes in the structural properties and energy beyond natural fluctuations. 


\subsection{In vacuum (or bad solvent)}

\subsubsection{Configuration change of PDMS during adsorption}

Fig.1 shows the simulation snapshots of the PDMS chains with $N=40$. Furthermore, it shows good adsorption during the simulation time on the silicon (111) surface. Interestingly, the sequent configurations present curved not straight, and they all present 2-D adsorption configuration from the side view when the single chain has adsorbed well on the surface. With time evolution, the chain configuration changes from initially isolated "random-coil" in a

(a)

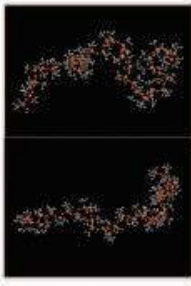

0ps (a)



$11 \mathrm{ps}$

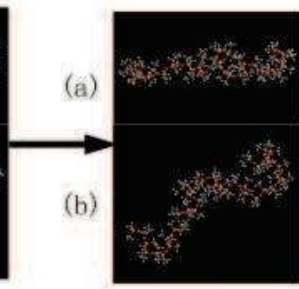

$14 \mathrm{ps}$

(a)

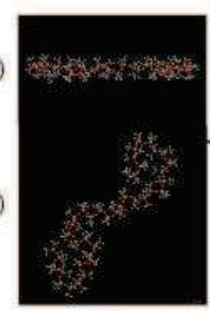

800 ps (b)

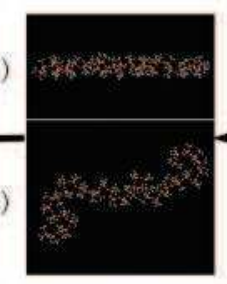

$279 \mathrm{ps}$

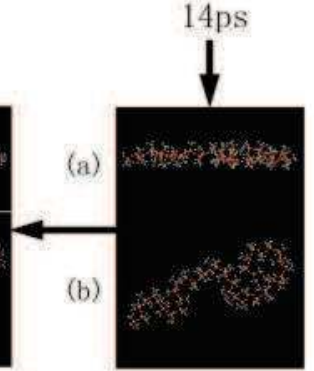

$99 \mathrm{ps}$

(a)

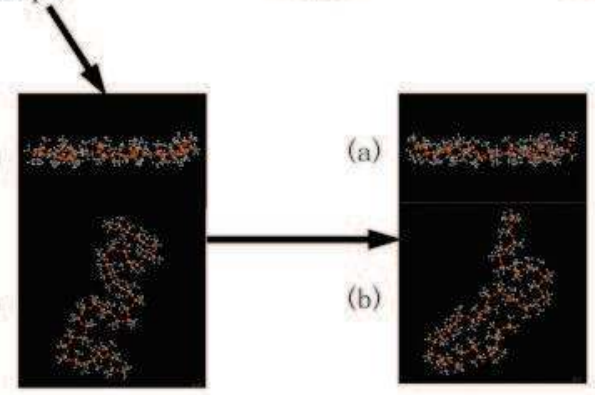

$1300 \mathrm{ps}$

5 ns

Fig. 1. We take the case of $N=40$ for example, extract the snapshots of the configurations during its adsorption process.

vacuum to a compact form adsorbed on the surface. In the first several hundred picoseconds, chain adsorption occurs accompanied by diffusion. When the energy dynamically reaches constant, the chain dynamics is mainly dominated by the diffusion process. Fig.2 shows the simulation snapshots of the PDMS chains with $N=10,40,50$ and 60 in equilibrium, but the configurations of longer chain like $N=50$ and 60 present partial arched part. However, 
it shows good adsorption of no matter short or long PDMS single chain on this silicon surface. Interestingly, these configurations present curved, especially the long chains. Finally, they all present 2-D adsorption configuration from the side view when they reach both thermodynamic and kinetic equilibrium state. To show the chain configuration change during

(a)

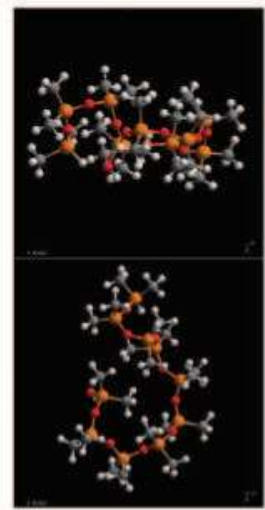

$\mathrm{N}=10$

(a)

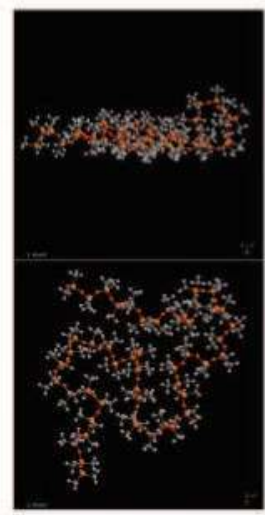

$\mathrm{N}=50$ (a)

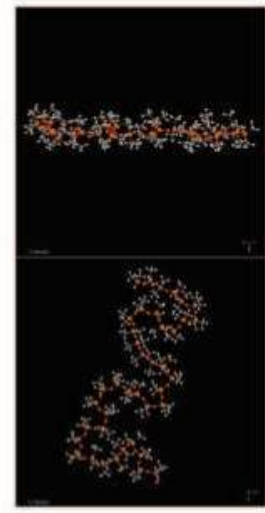

$\mathrm{N}=40$

(a)

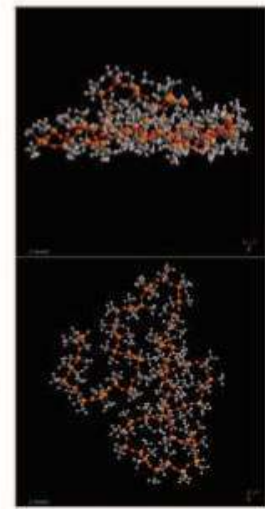

$\mathrm{N}=60$

Fig. 2. Snapshots for PDMS chains adsorbed on the silicon surface with $N=10,40,50$ and 60 are displayed. The gray color denotes carbon and the white denotes hydrogen. Red denotes oxygen, orange denotes silicon.

the adsorption, we calculate the mean radius of gyration which is defined as

$$
<R_{g}>=\sqrt{<\frac{1}{A} \sum_{i=1}^{N}\left(r_{i}-r_{c m}\right)^{2}>}
$$

where $r_{i}$ and $r_{c m}$ denote the position vector of each atom in a chain and the center-of-mass for the whole chain, respectively, and $A$ represents the number of atoms. Fig.3 shows the change 
of calculated $\left\langle R_{g}>\right.$ with increasing $A$. We fit this curve with the first order exponential decay function as $R_{g}=A_{1} \exp \left(-x / t_{1}\right)+y_{0}$, therein $y_{0}=6.82 \pm 0.35, A_{1}=-9.62 \pm 0.55$ and $t_{1}=18.76 \pm 2.9$.. By seeing the simulation snapshots, we can find that all the PDMS chain are ultimately well-adsorbed on the silicon surface with 2-D configurations. It appears increasing trend of $\left\langle R_{g}\right\rangle$ during the increase in the $N$ values, then it's amplitude becomes smaller and smaller, especially for the longer chains. For the sake of characterizing the

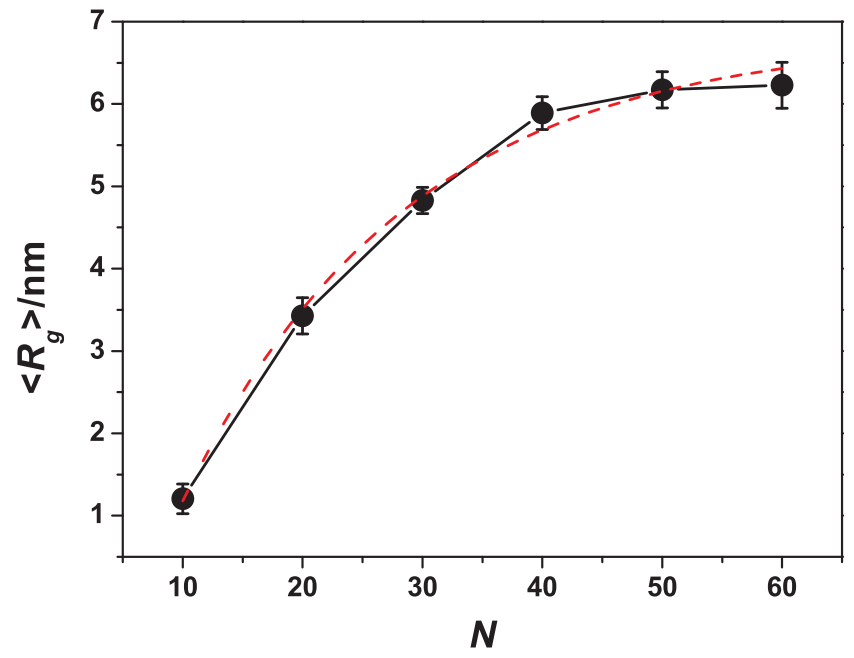

Fig. 3. The mean radius of gyration $R_{g}$ vs the chain length $N$. The symbols are MD simulation results. The error bars are the standard deviation measured in three parallel simulations.

anisotropic configuration of the polymer and interpreting the configuration change during adsorption, we calculate two ratios such as $\left(R_{x}^{2}+R_{y}^{2}\right) / R_{z}^{2}$ and $R_{\max } / R_{\min } . R_{x}, R_{y}$ and $R_{z}$ are the components in three principal directions of $R_{g} . R_{\max }$ and $R_{\min }$ correspond to the large and small magnitude between $R_{x}$ and $R_{y}$, respectively. The results are shown in Fig.4, panels $\mathrm{a}$ and $\mathrm{b}$. The component of $R_{g}$ along the $Z$ axis that is perpendicular to the surface is strongly reduced during the adsorption. The components of $R_{g}$ along the $X$ and $Y$ axes display significant increase due to the chain spreading on the surface. All the chains are well adsorbed on the surface and therefore present 2-D configurations. Thus $R_{x}$ and $R_{y}$ are larger and $R_{z}$ is very small.

\subsubsection{Diffusion of PDMS chain on the surface}

After the adsorption process, which is monitored by the interaction energy between the chain and the surface starting to fluctuate only around a constant value, the dynamics of PDMS is mainly dominated by the chain diffusion on the surface. We calculate the diffusion coefficients of the chains via the Einstein relation. Fig. 5 shows the change of $D$ with the varying degree of polymerization. There is apparent scaling between $D$ and $N$, that is, $D \sim N^{-3 / 2}$. The dependence of $D$ on $N$ can be explained by the change of interaction energy between the chain and surface with increasing $N$. This adsorption energy can be calculated via

$$
E_{\text {int }}=E_{\text {tot }}-\left(E_{\text {frozen }}+E_{\text {plane }}\right)
$$



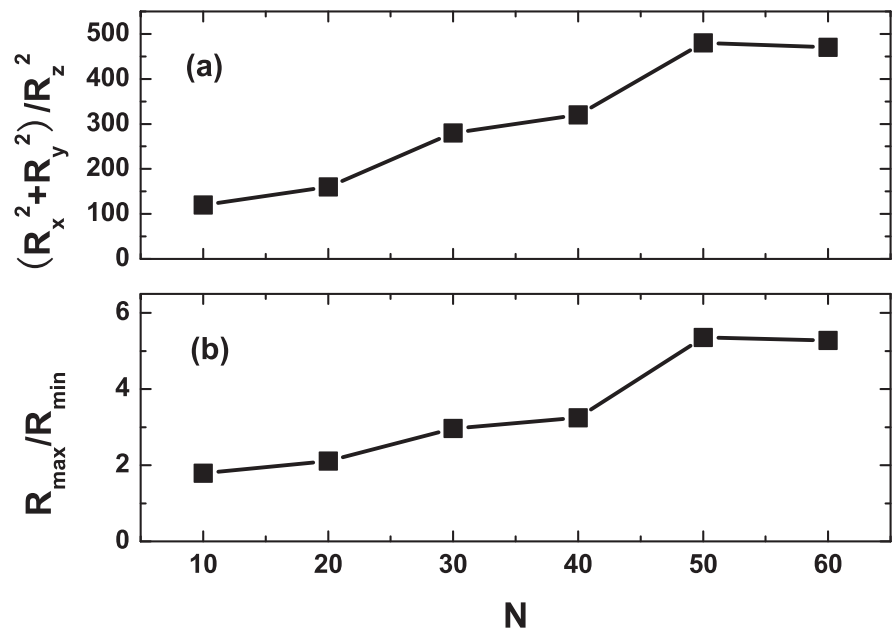

Fig. 4. These two ratios are calculated from the three principal components of $R_{g}$. Panel a shows the ratio of $\left(R_{x}^{2}+R_{y}^{2}\right) / R_{z}^{2}$ vs $N$ and panel b shows the ratio of $R_{\max } / R_{\min }$ vs $N$, where $R_{\max }$ denotes the larger one of $R_{x}$ or $R_{y}$, and $R_{\text {min }}$ denotes the other.

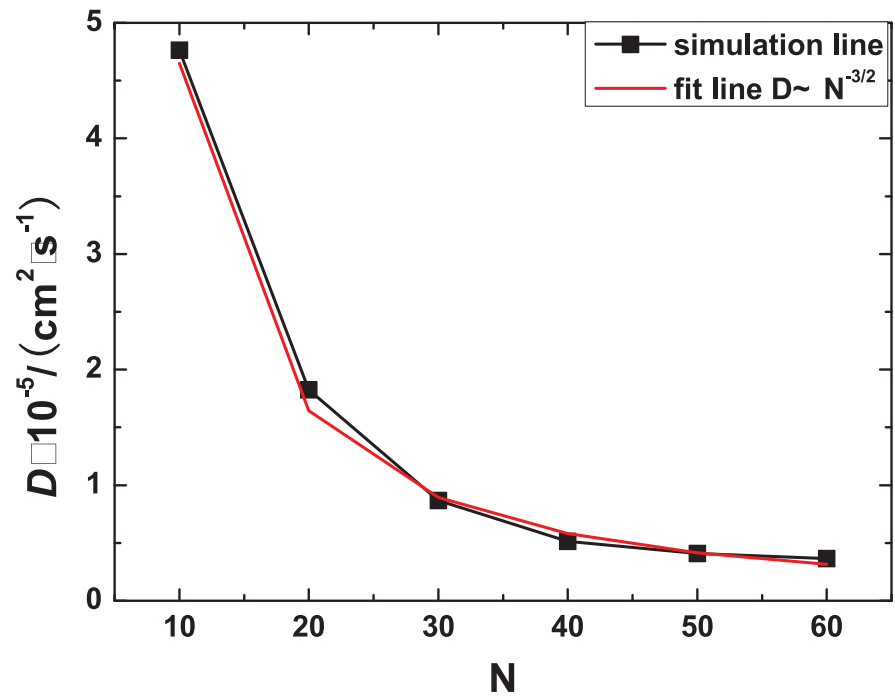

Fig. 5. Diffusion coefficients, $D$, are plotted against degree of polymerization of the PDMS chain, $N$.

where $E_{\text {tot }}$ is the potential energy of the chain plus the surface system in equilibrium, $E_{\text {frozen }}$ is the potential energy of the adsorbed chain isolated in a vacuum with the geometry unchanged, and $E_{\text {plane }}$ is the potential energy of the surface. Larger molecular configuration deformation allows for better adsorption of the chain onto the surface; however, this will break the intramolecular interaction which causes a free energy penalty. Therefore such an interaction competition results in all the adsorption energy with increasing $N$, which can be seen in Fig.6. 
We fit this curve with the linear function, and the average adsorption energy per segment $\left(E_{\text {int }} / N\right)$ is $-0.42 \mathrm{kcal} / \mathrm{mol}$.

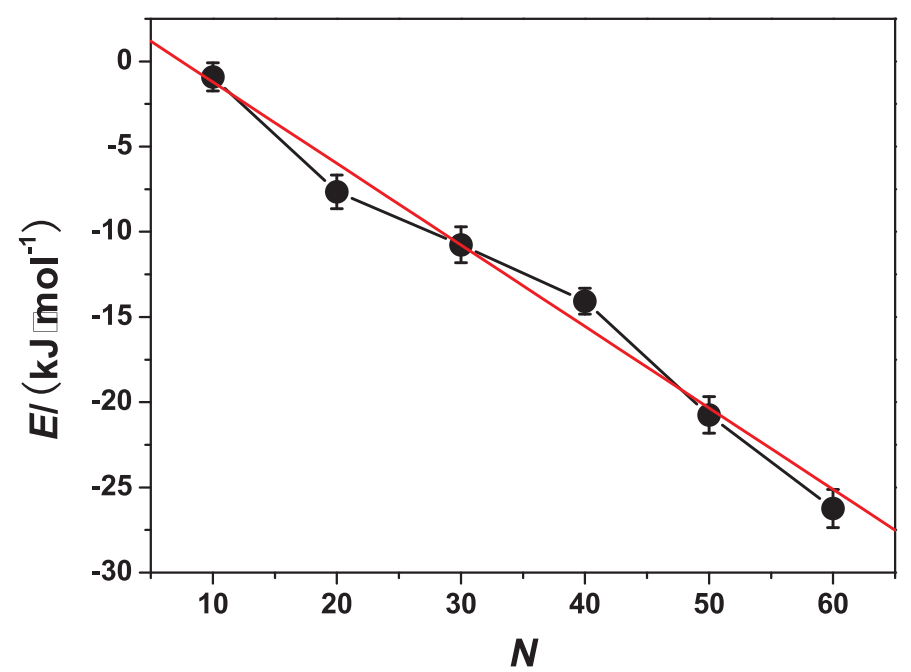

Fig. 6. Adsorption energy $E_{\text {int }}$ vs chain length $N$.

\subsection{In good solvent}

We change the dielectric constant to 78.0 to mimic the good solvent condition (41). Of course in this way the explicit solvation effects cannot be considered. Nevertheless, we can directly study the effects by simply changing the bad to the good solvent condition on the chain configurations and dynamics.

The PDMS chain also can be adsorbed onto the hydrophobic surface in good solvent environment, showing 2-D configurations, no matter how many monomers the chain contains. The adsorption takes place very fast and the equilibrium is attained around about 200 ps. Sequentially, 2-D chain configurations are always retained in the successive diffusion process. At last, the chain possesses the most stable configuration as one layer on the surface. For saving time and avoiding repetitious work, we have only chosen one special chains with the same length as $N=10$. Compared to the relative results in a vacuum, we can find that the contact area between the chain and the surface decrease, also the interaction energy increases, which results the data of $E_{\text {int }}$ and diffusion coefficients both increase. The results are shown in Table 1. Also the configurations are both changed: the configuration of $N=10$ turns from "coil pancake" to more extended configuration; and the configuration from the side view can be seen contains stacking part, which can be attributed to its lower diffusion coefficient value in good solvent compared with it in bad solvent. The equilibrium configuration of these two cases are present in Fig.7. In summary, it is clear that solvent effect has an important influence on the configurations and the dynamics of the hydrophobic-hydrophobic system from the discussion above. It can change not only the equilibrium chain configuration after the adsorption, but also the dynamics behavior of the chain on the surface. 



\section{$\mathrm{N}=10$}

Fig. 7. Snapshots for PDMS chains adsorbed on the silicon Surface with $N=10$ are displayed in bad (in vacuum) and good (in solution) solvent environment, respectively. The gray color denotes carbon and the white denotes hydrogen. Red denotes oxygen, orange denotes silicon.

\begin{tabular}{l|ll}
\hline & $E_{\text {int }}(\mathrm{kcal} / \mathrm{mol})$ & $D\left(\mathrm{~cm}^{2} / \mathrm{s}\right)$ \\
\hline in vacuum & -0.91 & $4.76 \times 10^{-5}$ \\
in good solvent & -0.85 & $7.42 \times 10^{-5}$ \\
\hline
\end{tabular}

Table 1. Details of Adsorption Energy and Diffusion Coefficient for the Adsorption Behavior of $N=10$ PDMS Single Chain on Silicon surface.

\section{Conclusions}

In this paper, MD simulations are used to investigate the adsorption and diffusion behavior of a single flexible hydrophobic PDMS chain on a hydrophobic silicon surface. Because of their similar characteristics, the PDMS chains are all adsorbed well onto the surface and possess 2-D configurations.

In a vacuum (or bad solvent), the PDMS chains are well adsorbed onto the hydrophobic surface and displays 2-D configuration. The calculated results of mean radius of gyration and two ratios such as $\left(R_{x}^{2}+R_{y}^{2}\right) / R_{z}^{2}$ and $R_{\max } / R_{\min }$ manifest the chain length dependence of the adsorption configuration. The adsorption energies are linearly scaled with $N$, therein the average $E_{\text {int }} / N$ value is $-0.42 \mathrm{kcal} / \mathrm{mol}$. In addition, the data of their diffusion coefficient obey scaling law with $N^{-3 / 2}$ for the considered chain lengths, which is the same as polyethylene on Si (111) surface (45).

In good solvent, the PDMS chain also can be adsorbed very well on the surface and displays 2-D configuration, especially for the chains with less monomers; when the chains become longer, it would present quasi 2-D configurations, owing to its larger adsorption energy and larger diffusion coefficient in good solvent environment, compared with that in bad solvent 
environment with the same chain length. Owing to the decrease of adsorption energy and diffusion coefficients, the configuration of $N=10$ in good solvent changes to be more extended with less folded number, compared with that in a vacuum environment. The lower adsorption energy determines the larger diffusion coefficient, which means the higher diffusion rate.

The above results show that a single PDMS chain can be adsorbed onto the hydrophobic surface well. This is affected by the solvent conditions. By changing from the bad to good solvent, the chain can be worse adsorbed, which means it is good for desorption in good solvent environment, accordingly, it is good for adsorption in bad solvent enviroment. Thus by fine-tuning the solvent quality, one can manipulate the behavior of the chain.

\section{Acknowledgment}

The project is supported by the Science-Technology Foundation for Middle-aged and Young Scientist of Shandong Province (BS2010CL048), Shandong Province Higher School Science \& Technology Fund Planning Project (J10LA61) and Zaozhuang Scientific and Technological Project (200924-2).

\section{References}

[1] de Gennes, P.G.(1979) Scaling Concepts in Polymer Physics, Cornell University Press, Ithaca, NY.

[2] Doi, M.; Edwards, S.F. (1986) The theory of Polymer Dynamics, Clarendon Press, Oxford, UK.

[3] Maier, B.; Rädler, J.O. (1999) Conformation and Self-Diffusion of Single DNA Molecules Confined to Two Dimensions Phys. Rev. Lett., 82, 1911-1914.

[4] Maier, B.; Rädler, J.O. (2000) DNA on Fluid Membranes: A Model Polymer in Two Dimensions Macromolecules, 33, 7185-7194.

[5] Yethiraj, A. (2002) Polymer Melts at Solid Surfaces Adv. Chem. Phys., 121, 89-139.

[6] Sheiko, S.S.; Möller, M. (2001) Visualization of moleculesąła first step to manipulation and controlled response Chem. Rev., 101, 4099-4123.

[7] Granick, S. (2002) Kinetic and Mechanical Properties of Adsorbed Polymer Layers Eur. Phys. J. E., 9, 421-424.

[8] Granick, S.; Kumar, S.K.; Amis, E.J.; Antonietti, M.; Balazs, A.C.; Chakraborty, A.K.; Grest, G.S.; Hawker, C.; Janmey, P.; Kramer, E,J.; Nuzzo, R.; Russell, T.P.; Safinya, C,R. (2003) Macromolecules at Surfaces: Research Challenges and Opportunities from Tribology to Biology J. Polym. Sci. Part B: Polym. Phys., 41, 2755-2793.

[9] Haynes, C.A.; Norde, W. (1994) Globular Proteins at Solid/Liquid Interfaces Colloids Surf. B., 2, 517-566.

[10] Malmsten, M. (1998) Formation of Adsorbed Protein Layers J. Colloid Interface Sci., 207, 186-199.

[11] Decher, G. (1997) Fuzzy Nanoassemblies: Toward Layered Polymeric Multicomposites Science, 277, 1232-1237.

[12] Esker, A.R.; Mengel, C.; Wegner, G. Ultrathin Films of a Polyelectrolyte with Layered Architecture (1998) Science, 280, 892-895.

[13] Caruso, F.; Caruso, R.A.; Mohwald, H. (1998) Nanoengineering of Inorganic and Hybrid Hollow Spheres by Colloidal Templating Science, 282, 1111-1114. 
[14] Shiratori, S.S.; Rubner, M.F. (2000) pH-Dependent Thickness Behavior of Sequentially Adsorbed Layers of Weak Polyelectrolytes Macromolecules, 33, 4213-4219.

[15] Clark, S.L.; Hammond, P.T. (1998) Engineering the Microfabrication of Layer-by-Layer Thin Films Adv. Mater., 10, 1515-1519.

[16] Husemann, M.; Morrison, M.; Benoit, D.; Frommer, J.; Mate, C.M.; Hinsberg, W.D.; Hedrick, J.L.; Hawker, C.J. (2000) Manipulation of Surface Properties by Patterning of Covalently Bound Polymer Brushes J. Am. Chem. Soc., 122, 1844-1845.

[17] Xia, Y.N.; Whitesides, G.M. (1998) Soft lithography Annu. Rev. Mater. Sci., 28, 153-184.

[18] Huck, W.T.S.; Strook, A.D.; Whiteside, G.M. (2000) Synthesis of Geometrically Well Defined, Molecularly Thin Polymer Films Angew. Chem. Int. Ed., 39, 1058-1061.

[19] Sukhishvili, S.A.; Chen, Y.; Müller, J.D.; Gratton, E.; Schweizer, K.S.; Granick, S. (2000) Materials science: Diffusion of a polymer pancake Nature, 406, 146-146.

[20] Bae, S.C.; Xie, F.; Jeon, S.; Granick, S. (2001) Single isolated macromolecules at surfaces Curr. Opin. Solid. State. Mater., 5, 327-332.

[21] Sukhishvili, S.A.; Chen, Y.; Müller, J.D.; Gratton, E.; Schweizer, K.S.; Granick, S. (2002) Surface Diffusion of Poly(ethylene Glycol) Macromolecules, 35, 1776-1784.

[22] Zhao, J.; Granick, S. (2004) Polymer lateral diffusion at the solid-liquid interface J. Am. Chem. Soc., 126, 6242-6243.

[23] Milchev, A.; Binder, K. (1996) Static and Dynamic Properties of Adsorbed Chains at Surfaces: Monte Carlo Simulation of a Bead-Spring Model Macromolecules, 29, 343-354.

[24] Azuma, R.; Takayama, H. (1999) Diffusion of single long polymers in fixed and low density matrix of obstacles confined to two dimensions J. Chem. Phys., 111, 8666-8671.

[25] Falck, E.; Punkkinen, O.; Vattulainen, I.; Ala-Nissila, T. (2003) Dynamics and scaling of two-dimensional polymers in a dilute solution Phys. Rev. E., 68, 050102.

[26] Cavallo, A.; Müller, M,.; Binder, K. (2005) Unmixing of Polymer Blends Confined in Ultrathin Films: Crossover between Two-Dimensional and Three-Dimensional Behavior J. Phys. Chem. B, 109, 6544-6552.

[27] Wang, Y.; Mattice, W.L. (1994) A Comparison between Monte Carlo Simulation and the Scheutjens-Fleer Mean-Field Lattice Theory Langmuir, 10, 2281-2288.

[28] Milchev, A.; Binder, K. (1997) Dewetting of thin polymer films adsorbed on solid substrates: A Monte Carlo simulation of the early stages J. Chem. Phys., 106, 1978-1989.

[29] Pandey, R.B.; Milchev, A.; Binder, K. (1997) Semidilute and Concentrated Polymer Solutions near Attractive Walls: Dynamic Monte Carlo Simulation of Density and Pressure Profiles of a Coarse-Grained Model Macromolecules, 30, 1194-1204.

[30] Varnik, F.; Baschnagel, J.; Binder, K.; Mareschal, M. (2003) Confinement effects on the slow dynamics of a supercooled polymer melt: Rouse modes and the incoherent scattering function Eur. Phys. J. E., 12, 167-171.

[31] Lin, Y.-C.; Müller, M.; Binder, K. (2004) Stability of thin polymer films: Influence of solvents J. Chem. Phys., 121(8), 3816-3828.

[32] Kim, D.-C.; Lu, W. (2006) Three-dimensional model of electrostatically induced pattern formation in thin polymer films Phys. Rev. B, 73, 035206.

[33] Desai, T.; Keblinski, P.; Kumar, S. (2006) Computer simulations of the conformations of strongly adsorbed chains at the solidíCliquid interface Polymer, 47, 722-727.

[34] Mu, D.; Lu, Z.-Y.; Huang, X.-R. (2008) The adsorption of poly (vinyl alcohol) on the hydroxylated $\beta$-cristobalite Molecular Simulation, 34(6), 611-618. 
[35] Mu, D.; Lu, Z.-Y.; Huang, X.-R.; Sun, C.-C. (2008) Ordered Adsorption of Polyethylene on Hydroxylated $\beta$ Cristobalite (100) Surface Chem. J. Chinese Universities, 29(10), 2065-2069.

[36] Mu, D.; Zhou, Y.-H. (2011) Molecular Dynamics Simulation of the Adsorption and Diffusion of a Single Hydrophobic Polymer Chain on a Hydrophobic Surface Acta Phys. -Chim. Sin., 27(2), 374-378.

[37] Sun, H. (1998) COMPASS: An ab Initio Force-Field Optimized for Condensed-Phase Applications - Overview with Details on Alkane and Benzene Compounds J. Phys. Chem. B, 102, 7338-7364.

[38] Sun, H.; Ren, P.; Fried, J.R. (1998) The compass force field: parameterization and validation for phosphazenes Comput. Theor. Polym. Sci., 8, 229-246.

[39] Allen, M.P.; Tildesley, D.J. (1987) Computer Simulation of Liquids, Clarendon, Oxford, UK.

[40] Berendsen, H.J.C.; Postma, J.P.M.; van Gunsteren, W.F.; Dinola, A.; Haak, J.R. (1984) Molecular-dynamics with coupling to an external bath J. Chem. Phys., 81, 3684-3690.

[41] Raffaini, G.; Ganazzoli, F. (2004) Molecular dynamics simulation of the adsorption of a fibronectin module on a graphite surface Langmuir, 20, 3371-3378.

[42] Rouse, P.E. (1953) A theory of the visco-elastic properties of dilute solutions of coiling polymers J. Chem. Phys., 21, 1272-1280.

[43] Shannon, S.R.; Choy, T.C. (1997) Dynamical Scaling Anomaly for a Two Dimensional Polymer Chain in Solution Phys. Rev. Lett., 79, 1455-1458.

[44] Ala-Nissila, T.; Herminghaus, S.; Hjelt, T.; Leiderer, P. (1996) Diffusive spreading of chainlike molecules on surfaces Phys. Rev. Lett., 76, 4003-4006.

[45] Mu, D.; Zhou, Y.-H. (2011) Adsorption and Diffusion Processes of Polyethylene on Silicon (111) Surface Studied by Molecular Dynamics Simulation J. Theor. Comput. Chem., 10(4), 411-421. 


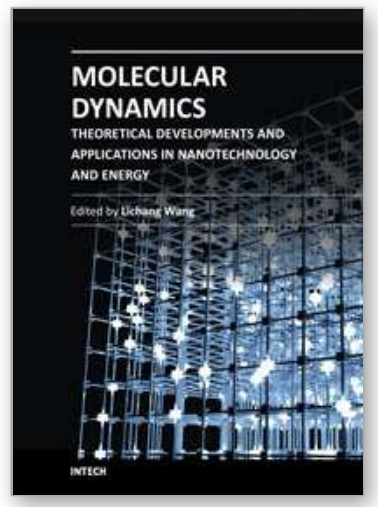

\author{
Molecular Dynamics - Theoretical Developments and Applications \\ in Nanotechnology and Energy \\ Edited by Prof. Lichang Wang
}

ISBN 978-953-51-0443-8

Hard cover, 424 pages

Publisher InTech

Published online 05, April, 2012

Published in print edition April, 2012

Molecular Dynamics is a two-volume compendium of the ever-growing applications of molecular dynamics simulations to solve a wider range of scientific and engineering challenges. The contents illustrate the rapid progress on molecular dynamics simulations in many fields of science and technology, such as nanotechnology, energy research, and biology, due to the advances of new dynamics theories and the extraordinary power of today's computers. This first book begins with a general description of underlying theories of molecular dynamics simulations and provides extensive coverage of molecular dynamics simulations in nanotechnology and energy. Coverage of this book includes: Recent advances of molecular dynamics theory Formation and evolution of nanoparticles of up to 106 atoms Diffusion and dissociation of gas and liquid molecules on silicon, metal, or metal organic frameworks Conductivity of ionic species in solid oxides Ion solvation in liquid mixtures Nuclear structures

\title{
How to reference
}

In order to correctly reference this scholarly work, feel free to copy and paste the following:

Dan Mu and Jian-Quan Li (2012). A Study of the Adsorption and Diffusion Behavior of a Single Polydimethylsiloxane Chain on a Silicon Surface by Molecular Dynamics Simulation, Molecular Dynamics Theoretical Developments and Applications in Nanotechnology and Energy, Prof. Lichang Wang (Ed.), ISBN: 978-953-51-0443-8, InTech, Available from: http://www.intechopen.com/books/molecular-dynamicstheoretical-developments-and-applications-in-nanotechnology-and-energy/a-study-of-the-adsorption-anddiffusion-behavior-of-a-single-polydimethylsiloxane-chain-on-a-silicon

\section{INTECH}

open science | open minds

\section{InTech Europe}

University Campus STeP Ri

Slavka Krautzeka 83/A

51000 Rijeka, Croatia

Phone: +385 (51) 770447

Fax: +385 (51) 686166

www.intechopen.com

\section{InTech China}

Unit 405, Office Block, Hotel Equatorial Shanghai

No.65, Yan An Road (West), Shanghai, 200040, China 中国上海市延安西路65号上海国际贵都大饭店办公楼405单元

Phone: +86-21-62489820

Fax: $+86-21-62489821$ 
(C) 2012 The Author(s). Licensee IntechOpen. This is an open access article distributed under the terms of the Creative Commons Attribution 3.0 License, which permits unrestricted use, distribution, and reproduction in any medium, provided the original work is properly cited. 